\title{
Mechanisms of tricuspid regurgitation in patients with hypoplastic left heart syndrome undergoing tricuspid valvuloplasty
}

\author{
Victor Bautista-Hernandez, MD, PhD, ${ }^{\mathrm{a}}$ David W. Brown, MD, ${ }^{\mathrm{b}}$ Hugo Loyola, BSc, ${ }^{\mathrm{a}}$ Patrick O. Myers, MD, \\ Michele Borisuk, RN, ${ }^{\mathrm{a}}$ Pedro J. del Nido, MD, ${ }^{\mathrm{a}}$ and Christopher W. Baird, MD ${ }^{\mathrm{a}}$
}

\begin{abstract}
Objectives: Tricuspid regurgitation (TR) remains a risk factor for morbidity and mortality through staged palliation in patients with hypoplastic left heart syndrome (HLHS). Reports on the mechanisms associated with TR in patients with HLHS are limited. Thus, we sought to describe our experience with tricuspid valve (TV) repair in these patients, focusing on the mechanisms of TR and corresponding surgical techniques.
\end{abstract}

\begin{abstract}
Methods: We performed a retrospective single-center review (January 2000 to December 2012) of patients with HLHS undergoing TV repair and completing Fontan circulation. We evaluated the pre- and postoperative echocardiograms, intraoperative findings, and surgical techniques used.
\end{abstract}

\begin{abstract}
Results: A total of 53 TV repairs were performed in 35 patients with HLHS completing staged palliation. TV repairs were performed at stage II in 15, between stage II and III in 4, at stage III in 27, and after stage III in 7. The surgical techniques for valvuloplasty included annuloplasty (38\%), anteroseptal (AS) commissuroplasty $(66 \%)$, anterior papillary muscle repositioning $(11 \%)$, multiple commissuroplasties $(9 \%)$, septal-posterior commissuroplasty $(9 \%)$, and fenestration closure $(4 \%)$. The predominant jet of TR emanated along the AS commissure in $68 \%$ of the cases. All patients survived the procedure and were discharged. Preoperative echocardiography showed a dilated TV annulus on the lateral dimension, anteroposterior dimension, and area that was significantly reduced after TV repair $(P<.0001)$. The preoperative mean TR, as assessed by lateral $(P=.002)$ and anteroposterior $(P=.005)$ vena contracta, was also significantly reduced after TV repair. TV repair did not significantly affect right ventricular systolic function immediately after surgery $(P=.17)$ or at the most recent follow-up visit $(P=.52)$. Patients with anterior leaflet prolapse were at increased risk of worse outcomes, including moderate or greater right ventricular dysfunction $(P=.02)$. Patients requiring reoperation for TV repair were younger $(6.3$ vs 28.1 months, $P<.0001)$ at the initial operation. One patient died of heart failure. Freedom from TV replacement and transplant-free survival were both $97 \%$ at the most recent follow-up point.
\end{abstract}

Conclusions: TR in patients with HLHS commonly emanates from the AS commissure. The associated mechanisms are often annular dilatation and anterior leaflet prolapse. Preoperative anterior leaflet prolapse was associated with worse outcomes. Annuloplasty, closure of the AS commissure, and repositioning of the anterior papillary muscle are effective in addressing TR in the short- and mid-term in this challenging population. (J Thorac Cardiovasc Surg 2014;148:832-40)

The outcomes of patients with hypoplastic left heart syndrome (HLHS) have improved dramatically during the past 2 decades. ${ }^{1-3}$ However, tricuspid regurgitation (TR) remains a risk factor for adverse outcomes in this challenging population. ${ }^{4-7}$ Despite significant TR in $25 \%$ of survivors through staged palliation, few data exist on

From the Departments of Cardiovascular Surgery ${ }^{\mathrm{a}}$ and Cardiology, ${ }^{\mathrm{b}}$ Boston Children's Hospital, Harvard Medical School, Boston, Mass.

Disclosures: Authors have nothing to disclose with regard to commercial support.

Read at the 94th Annual Meeting of The American Association for Thoracic Surgery, Toronto, Ontario Canada, April 26-30, 2014.

Received for publication April 24, 2014; revisions received June 23, 2014; accepted for publication June 27, 2014

Address for reprints: Christopher W. Baird, MD, Department of Cardiovascular Surgery, Boston Children's Hospital, 300 Longwood Ave, Bader 273, Boston, MA 02115 (E-mail: Chris.Baird@ cardio.chboston.org).

$0022-5223 / \$ 36.00$

Copyright (c) 2014 by The American Association for Thoracic Surgery

http://dx.doi.org/10.1016/j.jtcvs.2014.06.044 the underlying pathophysiology of TR in patients with HLHS. The reported mechanisms of TR in HLHS are complex and multifactorial and include structural abnormalities of the tricuspid valve (TV) and functional causes such as right ventricular (RV) dysfunction and dilatation of the TV annulus. ${ }^{8}$ Moreover, increasing age, with geometric changes of the annulus, leaflet prolapse, papillary muscle (PM) displacement, and subsequent leaflet tethering have been associated with TR in patients with HLHS. ${ }^{9,10}$

Several reports have suggested that TV repair decreases the severity of TR and could improve the outcomes in patients with HLHS. ${ }^{11-14}$ De Vega type annuloplasty, selective annuloplasty, cleft closure, chordal shortening, posterior leaflet obliteration, and commissure closure have been used to repair the TV in this population; however, no standard surgical approach exists. Recent advances in echocardiography and the understanding of the 


$$
\begin{aligned}
& \text { Abbreviations and Acronyms } \\
& \text { AP }=\text { anteroposterior } \\
& \text { AS }=\text { anteroseptal } \\
& \text { HLHS }=\text { hypoplastic left heart syndrome } \\
& \text { IQR }=\text { interquartile range } \\
& \text { PM }=\text { papillary muscle } \\
& \text { RV }=\text { right ventricular } \\
& \text { TR }=\text { tricuspid regurgitation } \\
& \text { TV } \quad \text { tricuspid valve }
\end{aligned}
$$

mechanisms of RV and TV function in the systemic circulation have made it possible to systematically evaluate the TV preoperatively with the aim of tailoring the surgical technique to specific anatomic abnormalities. Nevertheless, significant discrepancies between the echocardiographic and surgical findings have been reported and have complicated this approach. ${ }^{11}$

The purpose of our study was to evaluate the underlying echocardiographic mechanisms of TR in patients with HLHS completing staged palliation and correlate those with the efficacy and durability of the surgical approach undertaken.

\section{METHODS}

A retrospective analysis was undertaken of all patients with HLHS completing staged palliation through the Fontan procedure at Boston Children's Hospital from January 2000 through December 2012. The cardiac surgery and cardiac intensive care unit databases, hospital medical records, data from the echocardiography laboratory, and information from referring cardiologists were reviewed. All patients were followed up until death, lost to follow-up, or January 2014. Patients with anatomic variants without a morphologic right ventricle as the systemic ventricle were excluded from the present study. It has been consistently reported in published studies that patients showing significant TR before the Norwood procedure are at high risk of morbidity and mortality and thus will not complete staged palliation. ${ }^{12}$ Because the main goal of our study was to evaluate the underlying mechanisms of TR through follow-up of patients completing staged palliation and because TV plasty was not routinely performed during the early period of our study, we excluded patients who had undergone TV repair at stage I. The institutional review board approved the present study.

The pre- and postoperative echocardiograms were reviewed, and the parameters of interest were measured by a single experienced echocardiographer (D.W.B.). The existence and degree of TR was assessed with echocardiography using the 2-dimensional lateral and anteroposterior (AP) width of the vena contracta in the orthogonal planes and were categorized as none or trivial $(0-0.20 \mathrm{~cm})$, mild $(0.20-0.40 \mathrm{~cm})$, moderate $(0.40$ $0.60 \mathrm{~cm})$, or severe $(>0.60 \mathrm{~cm})$. In addition, TR was subjectively expressed as 0 (none or trivial), 1 (mild), 2 (moderate), or 3 (severe). The location of the regurgitant jet from the en face views was recorded. The mechanism of regurgitation was ascertained by noting the functional and structural abnormalities, such as valve morphology, leaflet prolapse, leaflet restriction, clefts, and ruptured chord. Prolapse was defined as any leaflet tissue above the plane of the tricuspid annulus in systole. The TV area and AP and lateral diameters were measured, and the Z-scores based on the patients' body surface area were calculated using our own echocardiography laboratory's database. ${ }^{15} \mathrm{RV}$ function was measured by subjective assessment and categorized as 0 (none or trivial), 1 (mild), 2 (moderate), or 3 (severely depressed).
The operative descriptions of the TV repair were compared with the echocardiographic data. The surgical techniques used were according to surgeon preference. To assess the durability of the TV repair, the echocardiogram at the most recent follow-up visit was reviewed for the degree of TR and assessment of RV function.

Generally, the TV was inspected in patients with HLHS with equal or greater than mild to moderate TR at staged palliation. After opening the right atrium, the surgeon checked the valve morphology and tested the competence with saline. We have previously described different surgical techniques used for TV repair. ${ }^{13,14}$ In brief, simple annuloplasty and commissuroplasty were the 2 major techniques used in patients with annular dilatation and/or leaflet prolapse. Partial annuloplasty was performed with a single stitch similar to the De Vega annuloplasty. Less commonly, a prosthetic ring was used. Prolapsing leaflets were resuspended by approximation with interrupted fine polypropylene suture to the adjacent leaflet, thus closing the commissure and improving the linearity of the coaptation area. More recently, we have incorporated repositioning of the anterior PM of the TV toward the septum to improve ventricular sphericity and valve tethering. ${ }^{13,16}$ Less commonly used techniques included chordal shortening for chordal elongation and the use of artificial chords for rupture chords.

\section{Statistical Analysis}

If continuous variables were normally distributed, the mean \pm standard deviation values were used and the median and interquartile range (IQR) if not. The pre- and postoperative changes in the continuous variables, TV dimensions and vena contracta, were compared using a paired $t$ test. Associations between dichotomous variables were compared using Fisher's exact test. In the case of the normal assumption not meeting, the analysis of 3 independent variables was performed using the asymptotic Kruskal-Wallis test. For 2 independent variables, the asymptotic Wilcoxon MannWhitney rank sum test was used. The Kaplan-Meier curve was used to estimate the freedom of RV dysfunction. All test and graphs were performed using R software. ${ }^{17}$

\section{RESULTS}

From January 2000 through December 2012, 53 TV repairs were performed in 35 patients with HLHS who had completed staged palliation through the Fontan procedure. All patients had mild to moderate or greater TR preoperatively. TV repair was performed at stage II in 15 , between stage II and III in 4, at stage III in 27 , and after stage III in 7. The characteristics of TV repair are listed in Table 1. The median age at the initial TV repair was 23.20 months (IQR, 6.31-194.0). All patients were alive at discharge without TV reintervention. Seventeen patients required repeat TV repair at a median age of 36.50 months (IQR, 3.81-97.3), with an interval between repairs of 23.30 months (IQR, 18.4-68.7).

\section{Perioperative Echocardiography}

The preoperative echocardiograms showed the predominant jet of TR emanating along the anteroseptal (AS) commissure in $36(68 \%)$, centrally in $12(23 \%)$, and posteriorly in $5(8 \%)$ of the 53 procedures. Preoperative anterior TV leaflet prolapse was identified on the echocardiogram in 31 of the 53 procedures $(58 \%)$ and was the most common cause of TR. Preoperative echocardiography showed a dilated TV annulus at the lateral dimension, AP dimension, 
TABLE 1. Characteristics of patients with tricuspid regurgitation and timing of valve repair

\begin{tabular}{lc}
\hline \multicolumn{1}{c}{ Characteristic } & \multicolumn{1}{c}{ Value } \\
\hline Total patients & 35 \\
Total TV repair procedures & 53 \\
Etiology of TR & \\
AS commissure & $36(68)$ \\
Central & $12(23)$ \\
Posterior & $04(8)$ \\
Stage of TV repair & \\
Stage II (BDG) & $15(28)$ \\
Between stage II and III & $04(8)$ \\
Stage III (Fontan) & $27(51)$ \\
After stage III & $7(13)$ \\
Timing of repair (mo) & \\
Age at first TV repair & $23.2(6.31-194)$ \\
Interval between repairs & $23.3(18.4-68.7)$ \\
Age at second TV repair & $36.5(27.1-97.30)$ \\
\hline Data presented as n, n (\%), or median (interquartile range). TV, Tricuspid valve; $T R$, \\
tricuspid regurgitation; $A S$, anteroseptal; $B D G$, bidirectional Glenn.
\end{tabular}

and area that was significantly reduced after TV repair $(P<.0001)$. The preoperative mean TR assessed subjectively and by the lateral $(P=.0022)$ and AP $(P=.0058)$ vena contracta had also decreased significantly after TV repair. A reduction in the TV dimensions and vena contracta was also significant after multiple TV repairs. The preoperative and postoperative changes after the first and second TV plasty are listed in Table 2. TV repair did not significantly affect RV systolic function immediately after surgery $(P=.17)$ or at the most recent follow-up visit $(P=.5228$; Figure 1).

\section{Surgical Assessment}

The surgical techniques for valvuloplasty were AS commissuroplasty in 35 (66\%), anterior PM repositioning in 6
(11\%), multiple commissuroplasties in $5(9 \%)$, septalposterior commissuroplasty in $5(9 \%)$, and fenestration closure in $2(4 \%)$ of the 53 procedures. Additional annuloplasty was performed in 20 of the 53 cases (38\%). Echocardiography was more sensitive in assessing leaflet motion abnormalities (prolapsed or tethering) and annular dilatation than in depicting some structural abnormalities. All patients undergoing annuloplasty had had a dilated TV annulus preoperatively. Prolapse of the anterior leaflet was detected by echocardiography in 31 of the 35 patients $(89 \%)$ who underwent AS commissuroplasty. PM repositioning was used in our series in 4 cases of anterior leaflet prolapse and 2 of predominant posterior leaflet-related regurgitation. However, 10 patients in our series underwent cleft $(\mathrm{n}=8)$ and fenestration $(\mathrm{n}=2)$ closure of previously undetected structural abnormalities.

\section{Late Follow-up}

The median follow-up period after 1 or multiple TV repairs was 38.18 months (IQR, 0.29-149.80) and 17.06 months (IQR, 0.19-138.3), respectively. More than $1 \mathrm{TV}$ repair was required in 17 patients $(48 \%)$ at a median interval of 23.20 months (IQR, 0.49-68.7). The median age at the first repair for patients requiring repeat repair was 6.38 months (IQR, 3.32-28.80). The median age was 28.1 months (range, 4.6-194) for those undergoing a single TV procedure $(P<.0001)$. Two patients underwent a third repair, one with annuloplasty ring adjustment and one with additional commissuroplasty.

Freedom from TV replacement and transplant-free survival were both $97 \%$ (34 of 35 patients) at the latest follow-up point. One patient, who had undergone multileaflet TV repair and a previous attempt at repair during stage III, required TV replacement. One patient died with moderate TR and severe RV dysfunction, who had undergone TV repair at stage III. Excluding the patient who died and the

TABLE 2. Changes in tricuspid valve dimensions, area, and vena contracta after first and second valvuloplasty

\begin{tabular}{|c|c|c|c|c|c|c|}
\hline \multirow[b]{2}{*}{ Tricuspid valve } & \multicolumn{3}{|c|}{ First tricuspid valvuloplasty } & \multicolumn{3}{|c|}{ Second tricuspid valvuloplasty } \\
\hline & Preoperative & Postoperative & $P$ value & Preoperative & Postoperative & $P$ value \\
\hline Patients (n) & 35 & 35 & & 17 & 17 & \\
\hline \multicolumn{7}{|l|}{ Annulus (Z-score) } \\
\hline Lateral & $4.07 \pm 1.7$ & $0.88 \pm 1.99$ & $<.0001$ & $2.59 \pm 1.84$ & $0.08 \pm 1.71$ & .0002 \\
\hline $\mathrm{AP}$ & $3.74 \pm 1.95$ & $0.36 \pm 1.6$ & $<.0001$ & $2.67 \pm 2.43$ & $0.29 \pm 1.62$ & .0058 \\
\hline Area (Z-score) & $6.34 \pm 2.7$ & $1.22 \pm 2.29$ & $<.0001$ & $4.09 \pm 3.11$ & $0.61 \pm 2.06$ & .0016 \\
\hline \multicolumn{7}{|l|}{ Vena contracta $(\mathrm{mm})$} \\
\hline Lateral & $0.47 \pm 0.19$ & $0.23 \pm 0.24$ & .0002 & $0.44 \pm 0.23$ & $0.17 \pm 0.24$ & .0015 \\
\hline $\mathrm{AP}$ & $0.41 \pm 0.17$ & $0.23 \pm 0.29$ & .0042 & $0.43 \pm 0.17$ & $0.20 \pm 0.10$ & .0004 \\
\hline \multicolumn{7}{|l|}{ TR grade (n) } \\
\hline 0 (none or trivial) & 0 & 15 & & 0 & 6 & \\
\hline 1 (mild) & $14 *$ & 16 & & $8 *$ & 10 & \\
\hline 2 (moderate) & 13 & 4 & & 6 & 1 & \\
\hline 3 (severe) & 8 & 0 & & 3 & 0 & \\
\hline
\end{tabular}

Data presented as mean \pm standard deviation, unless otherwise noted. $A P$, Anteroposterior; $T R$, tricuspid regurgitation. $*$ Mild to moderate TR. 


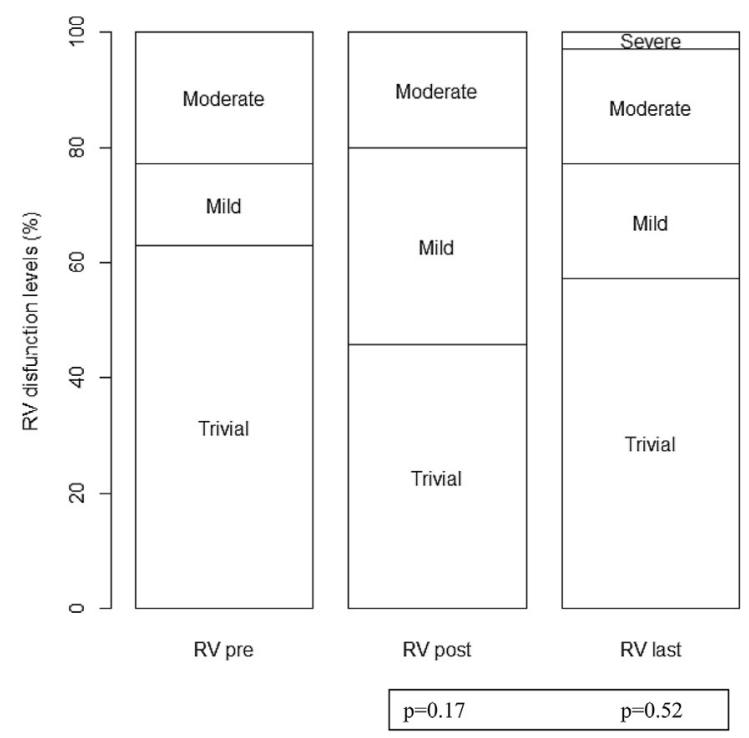

FIGURE 1. Changes in right ventricular $(R V)$ function from preoperatively (pre) to early postoperatively (post) to the most recent follow-up point (last) using 2-dimensional echocardiography.

patient requiring TV replacement, 5 of the 33 patients $(15 \%)$ showed significant TR (moderate or greater; Figure 2, A) and $7(21 \%)$ had significant RV dysfunction (moderate or greater) on the most recent follow-up echocardiogram (Figure 2, B).

No predictors of recurrent TR were identifiable when the data were analyzed by the preoperative mechanism of TR (AS commissure, central, posterior) and surgical technique used at repair (AS commissuroplasty, anterior PM repositioning, triple commissuroplasty, septal-posterior commissuroplasty, fenestration closure). However, the preoperative anatomy with an anterior TV leaflet prolapse was significantly associated with moderate or greater RV dysfunction at the most recent follow-up point $(P=.02$; Figure 2,C). Moreover, all 5 patients with significant TR at the most recent follow-up echocardiogram had had a dilated annulus preoperatively compared with the other patients and a prolapsed anterior TV leaflet.

\section{DISCUSSION}

Significant TR at birth is a well-known risk factor for early mortality after stage I palliation ${ }^{12}$ and is a common late finding, with approximately $25 \%$ of stage I survivors undergoing TV intervention within 10 years. ${ }^{8}$ In patients with HLHS, the TV functions as the systemic atrioventricular valve; therefore, anatomic abnormalities of the TV will have dramatic effects on morbidity and mortality. Despite TR being a significant risk factor in patients with HLHS, the underlying mechanisms of TR remain unclear. Therefore, no standard surgical approach has been widely accepted. In our series, we evaluated $53 \mathrm{TV}$ plasties in 35 patients with HLHS and found that the predominant jet of
TR emanated along the AS commissure in $68 \%$, centrally in $23 \%$, and posteriorly in $8 \%$. Furthermore, anterior leaflet prolapse was identified as the most common cause of TR preoperatively. Annular dilatation appeared as a secondary phenomenon. Patients with anterior leaflet prolapse were at increased risk of worse outcomes, including moderate or greater RV dysfunction $(P=.02)$ and recurrent TR at late follow-up. The patients requiring reoperation for TV repair were younger (6.3 vs 28.1 months, $P<.0001$ ) at the initial operation. One patient died during the follow-up period. Freedom from TV replacement and transplant-free survival were both $97 \%$ at the most recent follow-up visit.

The mechanism of TV regurgitation in HLHS is complex, multifactorial, and can be anatomic or functional. ${ }^{11,12}$ Furthermore, the actual contributing factors toward TR have not been consistently agreed on. ${ }^{11}$ When considering the mechanisms contributing to TR in patients with HLHS, one must consider the annulus, leaflets, chordal structures, and ventricle. Abnormalities of $\geq 1$ of these structures can be present at birth or can develop after stage I. Frequently occurring after the development of TR, additional annular dilatation and worsening TR can occur from increased volume loading, which alters the RV and PM geometry. Commonly in HLHS, the anterior leaflet of the TV will be prolapsed, the septal leaflet will be tethered, and the annulus will be dilated. During the past decade, surgeons have recognized the importance of directly addressing the mechanisms of TR in all stages of palliation. A number of techniques have been reported ${ }^{13,14,16,18-21}$ that initially focused on addressing annular dilatation. Bharucha and colleagues ${ }^{11}$ reported on the mechanisms of TR in 32 patients with HLHS and compared the echocardiographic and surgical findings. Compared with controls, the anterior leaflet was abnormal in $97 \%$ of cases, the septal leaflet in $91 \%$, and the posterior leaflet in $66 \%$. The abnormality most commonly producing regurgitation was a prolapsing anterior leaflet in 59\%, and annular dilatation was present in $91 \%$ of the patients. Although the mechanisms of TR were identified, it is important to note that the most common surgical techniques used were partial annuloplasty and commissuroplasty, which did not necessarily address all pathologic features. Kasnar-Samprec and colleagues ${ }^{22}$ reported on 18 of 90 consecutive patients with HLHS with significant TR before stage II. Similarly, all patients had a dilated annulus, the anterior leaflet was prolapsed in $61 \%$ of the patients, and septal leaflet tethering was present in $67 \%$. Unloading by the superior cavopulmonary anastomosis promoted RV remodeling but failed to improve TR. $^{22}$ In 2004, Ohye and colleagues ${ }^{19}$ analyzed the results of 28 patients with HLHS and significant TR after stage I. Successful TV repairs were predictive of preserved TV and RV function, with older and larger patients (age, 20 months, and weight, $8.7 \mathrm{~kg}$, vs age, 6.1 months, and weight, $4 \mathrm{~kg}$ ) having improved late outcomes. Partial annuloplasty 
Kaplan-Meier estimate of TR free

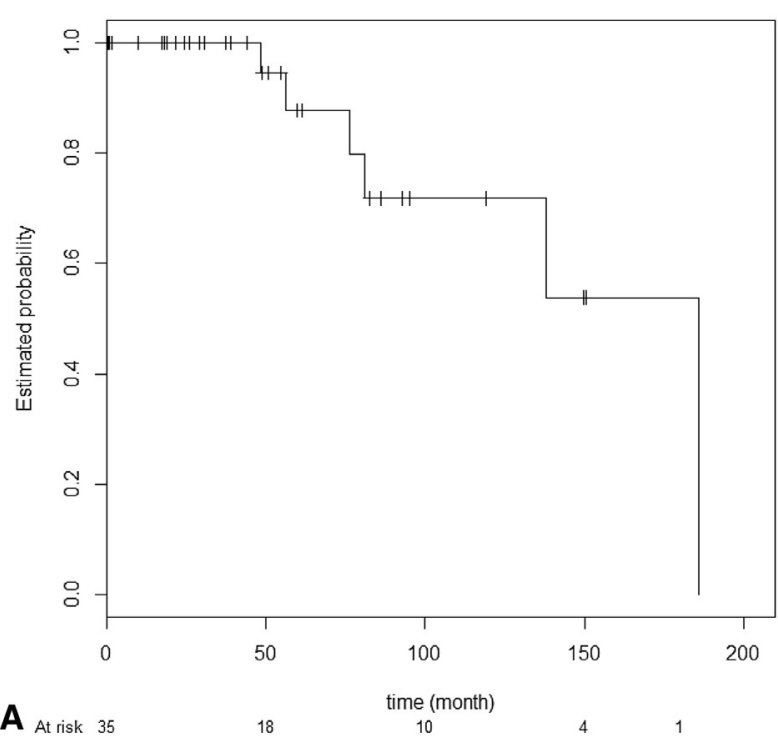

K-M estimate of RV dysfunction free

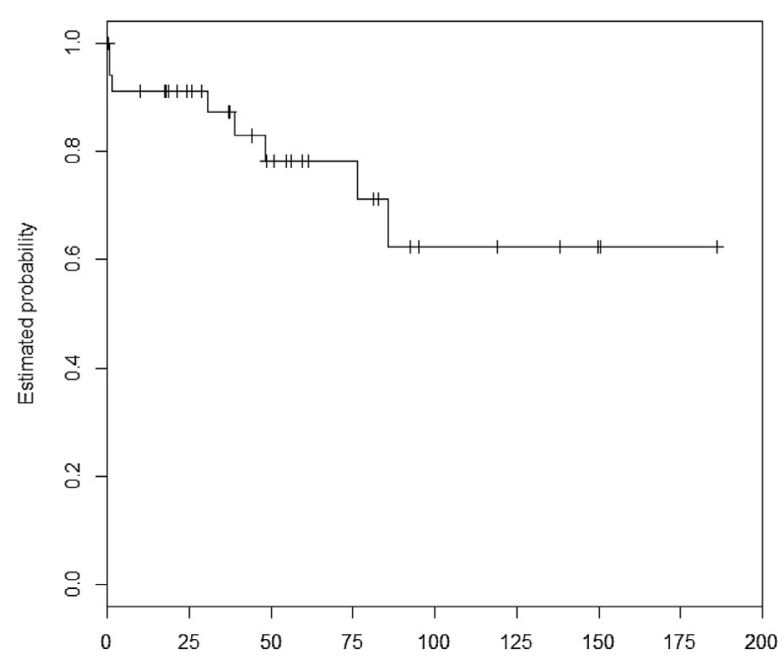

B

K-M estimate of RV dysfunction

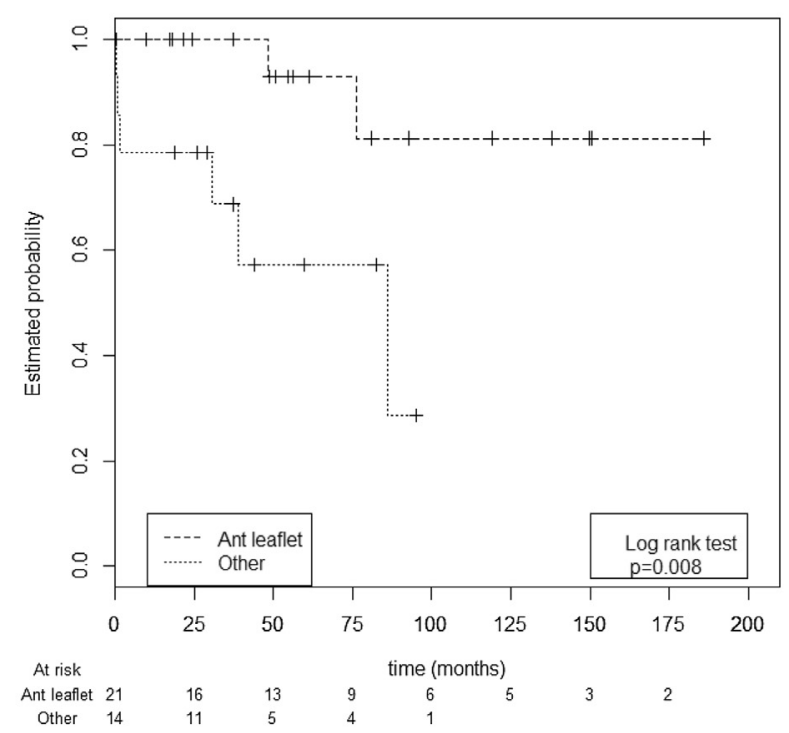

FIGURE 2. Kaplan-Meier ( $K-M)$ curves estimating freedom from (A) significant (moderate or greater) tricuspid regurgitation (TR), (B) right ventricular $(R V)$ dysfunction, and $(\mathrm{C})$ significant $\mathrm{RV}$ dysfunction by preoperative tricuspid valve anatomy (anterior [Ant] leaflet prolapse vs other anatomy).

to obliterate the posterior leaflet and form a bicuspid TV was the most common technique and proved to be the most effective, with improved outcomes. ${ }^{19-21}$ Ugaki and colleagues $^{23}$ also reported on 17 patients with HLHS, of whom $82 \%$ had a dilated annulus and central regurgitation. After posterior annuloplasty, the TR was improved, the RV end-diastolic area had decreased, fractional area change had occurred, and the coaptation length had improved. ${ }^{23}$ Although posterior annuloplasty can address TR at the level of the septal-posterior commissure, it does not address the regurgitation emanating from the AS commissure. In our series, to address anterior leaflet prolapse and/or TR through the AS commissure, we closed the AS commissure in $66 \%$ of the patients.

The long-term ability of the TV to support the systemic circulation is limited, because the TV is not designed to function with a circular annulus, such as occurs with RV dilatation. Nii and colleagues ${ }^{9}$ reported on TV annular function in 26 patients with HLHS at different stages of palliation using 3-dimensional echocardiography. The TV annulus was flattened, rather than saddle shaped, throughout ventricular systole, limiting septal-lateral contraction at the AS commissure, with resultant TR. ${ }^{9}$ They also found that annular dilatation and area change 
correlated with TR. The anterior TV leaflet was primarily involved in $63 \%$, with AS commissuroplasty and annuloplasty performed in $74 \%$ and $96 \%$, respectively. TV repair significantly reduced the annular measurements and TR without affecting RV function. ${ }^{9}$ Similarly, in our series, the most common preoperative finding was annular dilatation with an increased TV area and diameter. However, all patients with significant TR at the most recent follow-up visit had had a severely dilated TV annulus and prolapse of the anterior leaflet preoperatively.

Preservation of RV function is crucial to prevent morbidity and mortality in patients with HLHS. Controversial data exist regarding whether RV dysfunction and repeat TV repair are closely associated. In our series, we found no significant decrease in RV function after TV repair. RV dysfunction and dilatation can also have significant effects on the subvalvar components of the TV. In addition to reporting changes in annular shape, Nii and colleagues ${ }^{9}$ reported more lateral displacement of the anterior leaflet PM in patients with significant TR. They speculated that displacement of the anterior leaflet PM pulls the anterior leaflet from the septal leaflet, with resultant regurgitation. ${ }^{9}$ More recently, Takahashi and colleagues ${ }^{10}$ evaluated 35 patients with HLHS (10 after stage I, 12 after stage II, and 13 after stage III) using 2- and 3-dimensional echocardiography. They observed an enlarged TV area in those with HLHS compared with the control patients and significant, associated TR with leaflet tethering and/or prolapse. Similar to observations in adults with functional $\mathrm{TR},{ }^{24,25}$ tethering was related to lateral displacement of the supporting PM. They concluded that the TV annular dynamics and PM displacement are important associations with significant TR in patients with HLHS.

We have previously reported RV PM approximation as a novel technique for the repair of functional TR. PM repositioning was more effective than annuloplasty alone in reducing TR and improving ventricular sphericity and valve tethering. The reduction of the annular dimension was especially evident in the septal-lateral diameter. ${ }^{16}$ Moreover, our early clinical experience with this technique in children with TR and congenital heart disease showed less than mild TR in $88 \%$ of patients at a mean follow-up of 13 months. ${ }^{13}$ In the present series, we used PM repositioning in 6 patients, with only 1 having significant TR at the most recent follow-up visit. However, our experience with this technique was too small, with insufficient follow-up, for conclusions regarding its value.

\section{Study Limitations}

The limitations of the present study were primarily related to the retrospective nature of the analysis. The limited number of patients with HLHS and complex multifactorial nature of TR requires a cohort from multiple institutions for a prospective evaluation to precisely evaluate and correlate the preoperative echocardiographic and surgical findings and their relationship to specific surgical techniques and associated long-term outcomes. The analysis was based on the available 2-dimensional images. However, they were sufficient in all cases to analyze the TV dimensions and vena contracta. Additional, complementary information could be provided by 3 -dimensional echocardiography. There was also a risk of selection bias. TR is a well-known factor that avoids progression through staged palliation in those with HLHS; thus, we might not have captured patients with more severe forms of TR. However, the finding of a frequent common mechanism of TR in patients with HLHS completing Fontan circulation has potential importance for the surgical treatment of this challenging population.

\section{CONCLUSIONS}

TV repair in patients with HLHS after Fontan completion is feasible and can be accomplished in most patients, reducing TR and the TV dimensions without significant changes in RV function. The most common preoperative TV features were annular dilatation and anterior leaflet prolapse with regurgitation at the level of the AS commissure. Preoperative annular dilatation and anterior TV leaflet prolapse continue to be associated with worse outcomes. With increasing experience, a tailored, multilevel approach can be undertaken at the annulus, leaflets, PMs, or ventricle, depending on the underlying pathophysiologic mechanism of TR in this challenging population.

\section{References}

1. Norwood WI, Lang P, Hansen DD. Physiologic repair of aortic atresiahypoplastic left heart syndrome. N Engl J Med. 1983;308:23-6.

2. Tweddell JS, Hoffman GM, Mussatto KA, Fedderly RT, Berger S, Jacquis RD, et al. Improved survival of patients undergoing palliation of hypoplastic left hear syndrome: lessons learned from 115 consecutive patients. Circulation. 2002; 106(suppl I):I82-9.

3. Bautista-Hernandez V, Marx GR, Gauvreau K, Pigula FA, Bacha EA, Mayer JE et al. Resection of posterior coarctation shelf reduces neo-aortic obstruction in hypoplastic left heart syndrome. J Thorac Cardiovasc Surg. 2007;133:1540-6.

4. Barber G, Helton JG, Aglira BA, Chin AJ, Murphy JD, Pigott JD, et al. The significance of tricuspid regurgitation in hypoplastic left-heart syndrome. Am Heart J. 1998;116:1563-7.

5. Sano S, Huang SC, Kasahara S, Yoshizumi K, Kotani Y, Ishino K. Risk factors after the Norwood procedure using a right ventricle to pulmonary artery shunt. Ann Thorac Surg. 2009;87:178-85.

6. Reyes A II, Bove EL, Mosca RS, Kulik TJ, Ludomirsky A. Tricuspid valve repair in children with hypoplastic left heart syndrome during staged surgical reconstruction. Circulation. 1997;96(suppl II):II341-3.

7. Bautista-Hernandez V, Scheurer M, Thiagarajan R, Salvin J, Pigula FA, Emani S, et al. Right ventricle and tricuspid valve function at midterm after the Fontan operation for hypoplastic left heart syndrome: impact of shunt type. Pediatr Cardiol. 2011;32:160-6.

8. Elmi M, Hickey E, William WG, Van Arsdell GV, Caldarone CA, McCrindle BW. Long-term tricuspid valve function after Norwood operation. J Thorac Cardiovasc Surg. 2011;142:1341-7.

9. Nii M, Guerra V, Roman KS, Macgowan CK, Smallhorn JF. Three-dimensional tricuspid annular function provides insight into the mechanisms of tricuspid valve regurgitation in classic hypoplastic left heart syndrome. JAm Soc Echocardiogr. 2006;19:391-402. 
10. Takahashi K, Inage A, Rebeyka I, Ross D, Thompson RB, Mackie AS, et al. Realtime 3-dimensional echocardiography provides new insight into mechanisms of tricuspid regurgitation in patients with hypoplastic left heart syndrome. Circulation. 2012;120:1091-8.

11. Bharucha T, Honjo O, Seller N, Atlin C, Redington A, Caldarone CA, et al. Mechanisms of tricuspid regurgitation in hypoplastic left heart syndrome: a case-matched echocardiographic-surgical comparison study. Eur Heart J Cardiovasc Imaging. 2013;14:135-41

12. Sugiura J, Nakano T, Oda S, Ucui A, Ueda Y, Kado H. Effects of tricuspid valve surgery on tricuspid regurgitation in patients with hypoplastic left heart syndrome: a non-randomized series comparing surgical and non-surgical cases. Eur J Cardiothorac Surg. 2014;46:8-13.

13. Myers PO, Haruo Y, Baird C, Fynn-Thompson F, Emani S, del Nido PJ. Papillary muscle approximation to address tricuspid tethering: efficacy and safety from iatrogenic stenosis a case-control study. J Am Coll Cardiol. 2012;59:E762-72.

14. Bautista-Hernandez V, Myers PO, Loyola H, Marx GR, Bacha EA, Baird CW, et al. Atrioventricular valve annular remodeling with a bioabsorbable ring in young children. J Am Coll Cardiol. 2012;60:2256-816.

15. Lopez L, Colan SD, Frommelt PC, Ensing GJ, Kendall K, Younoszai AK, et al. Recommendations for quantification methods during the performance of a pediatric echocardiogram: a report from the Pediatric Measurements Writing Group of the American Society of Echocardiography Pediatric and Congenital Heart Disease Council. J Am Soc Echocardiogr. 2010;23:465-95.

16. Yamauchi H, Vasilyev NV, Marx GR, Loyola H, Padala M, Yoganathan AP, et al. Right ventricular papillary muscle approximation as a novel technique of valve repair for functional tricuspid regurgitation in an ex vivo porcine model. J Thorac Cardiovasc Surg. 2012;144:235-42.

17. R Core Team. R: a language and environment for statistical computing. Vienna, Austria: R Foundation for Statistical Computing; 2013. Available at: http:www. R-project.org/. Accessed April 15, 2014.

18. Kanter KR, Forbess JM, Fyfe DA, Mahle WT, Kirshborn PM. De Vega tricuspid annuloplasty for systemic tricuspid regurgitation in children with univentricular physiology. J Heart Valve Dis. 2004;13:86-90.

19. Ohye RG, Gomez CA, Goldber CS, Graves HL, Devaney EJ, Bove EL. Tricuspid valve repair in hypoplastic left heart syndrome. J Thorac Cardiovasc Surg. 2004; 127:465-72.

20. Ohye RG, Gomez CA, Goldberg CS, Graves HL, Devaney EJ, Bove EL. Repair of tricuspid valve in hypoplastic left heart syndrome. Cardiol Young. 2006; 16(suppl 3):21-6.

21. Dinh DC, Gurney JG, Donohue JE, Bove EL, Hirsch JC, Devaney EJ, et al. Tricuspid valve repair in hypoplastic left heart syndrome. Pediatr Cardiol. 2011;32:599-606.

22. Kasnar-Samprec J, Kuhn A, Horer J, Vogt M, Cleuziou J, Lange R, et al. Unloading of right ventricle by bidirectional superior cavopulmonary anastomosis in hypoplastic left heart syndrome patients promotes remodeling of systemic right ventricle but does not improve tricuspid regurgitation. I Thorac Cardiovasc Surg. 2012;144:1102-9.

23. Ugaki S, Khoo NS, Ross DB, Rebeyka IM, Adatia I. Tricuspid valve repair improves early right ventricular and tricuspid valve remodeling in patients with hypoplastic left heart syndrome. J Thorac Cardiovasc Surg. 2013;145:446-50.

24. Sukmawan R, Watabane N, Ogasawara Y, Yamaura Y, Yamamoto K, Wada N, et al. Geometric changes of tricuspid valve tenting in tricuspid regurgitation secondary to pulmonary hypertension quantified by a novel system with transthoracic real-time 3-dimensional echocardiography. J Am Soc Echocardiogr. 2007;20:470-6.

25. Fukuda S, Gilinov AM, Song JM, Daimon M, Kongsaerepong V, Thomas D, et al. Echocardiographic insight into atrial and ventricular mechanisms of functional tricuspid regurgitation. Am Heart J. 2006;152:1208-14.

\section{Discussion}

Dr Jennifer Hirsch-Romano (Ann Arbor, Mich). Thank you for an excellent presentation and demonstration of very good outcomes for a challenging population. TV regurgitation certainly is the bane of existence for surgeons caring for patients with HLHS, and, as your results demonstrate, this can be a very difficult lesion to address. You had excellent short-term outcomes but demonstrated that TR often recurs late for these patients.

We have studied TV repairs quite extensively in our own patients, and, as a result of those analyses, we have devised a slightly one-size-fits-all approach. We have found that in our hands obliterating the posterior leaflet as our form of annuloplasty has been the most effective, aside from addressing any obvious leaflet structural abnormalities.

From your study findings, it looks like really AS commissuroplasty, plus or minus annuloplasty, has been the most effective for your patients. Have you noticed since the completion of your study that there has been a change in approach to these patients with a more unified surgical plan?

Dr Bautista-Hernandez. These results are very recent. We conducted this study until January 2014, so as far as I can tell, we did not change our practice because of this study so far.

We are very aware of your previous publications from your institution on TR, with very good outcomes, too; however, our concern is that with the bicuspidization of the tricuspid valve, I do not know how effective that will be to address the TR if the jet comes from the AS commissure, which is what we have seen in our study.

Dr Hirsch-Romano. Specifically, how do you perform your annuloplasty? Do you perform it with a sizer, and do you use absorbable or nonabsorbable suture? This is a 2-pronged question, because Dr del Nido is also in the audience, what are your thoughts on bioabsorbable rings in the future for performing annuloplasty for these patients.

Dr Bautista-Hernandez. That is a very good point. I did not comment on the technique we used for annuloplasty. In our series, we have the De Vega annuloplasty, we have partial annuloplasty, and a few patients received rings. One of them had a resorbable ring placed and another two had just regular rings. Thus, really, it has been the surgeon's preference so far. So that is what I can tell.

Dr Hirsch-Romano. When you perform your annuloplasty, do you use a sizer at all or is it just based on an "eyeball", measurement?

Dr Bautista-Hernandez. It is a combination of both. We do measure the TV annulus, but we have not regularly downsized the valve to a $\mathrm{Z}$-score of 0 , for instance, because the $\mathrm{Z}$-scores were determined from measurements from regular biventricular hearts and, thus, are really not meant for patients with HLHS.

Dr Hirsch-Romano. In regard to the timing of your TV repair, we quite often try to address any element of TV regurgitation before the Fontan operation, partially based on data showing that the length of cardiopulmonary bypass and crossclamp time at Fontan can affect the long-term outcomes. Can you talk a little bit about what your decision tree is for the timing of repair.

Dr Bautista-Hernandez. As you are very well aware, we usually perform the intracardiac tunnel Fontan operation, so those patients require an aortic crossclamp and opening of the right atrium, so you have the TV right there. Thus, we usually take advantage of this approach to repair the TV.

Dr Hirsch-Romano. So, for patients with mild to moderate TR, would they tend to wait until the Fontan rather than undergoing TV repair earlier?

Dr Bautista-Hernandez. I mean it depends on every patient. In the present series, we have some patients who underwent the repair at stage II, between stage II and III, and even some of them underwent the repair later on after the Fontan, so it is a tailored approach. But at the time of the Fontan, because we have the TV there, we try to fix it if we see more than mild to moderate TR.

Dr Harald L. Lindberg (Oslo, Norway). That was a very nice presentation. I just wonder, the reason for the TR, have you made 
any correlation study of the timing of the second stage to the timing of volume overload of the heart, if that is correlated to the occurrence of late TR? I think with your numbers that would be possible to do.

Dr Bautista-Hernandez. That would be possible to do. But when we decided to do this study, we wanted to focus on the timing of stage III, precisely one of the reasons was that. Many reports have been published stating that perhaps reduction of the volume overload of the right ventricle at stage II can reduce the amount of TR. However, the data are controversial, so we tend to repair whenever we have significant TR at any stage.

Dr Emile Bacha (New York, NY). Victor, great presentation. Very good results, but disappointing at the same time, because the RV function was not affected. When you think about what we try to do, ultimately, we are trying to preserve or even improve $\mathrm{RV}$ function. I am as aggressive as the surgeons in Boston with repairing TVs in patients with HLHS, but it seems to me we are attacking a symptom and not the origin of the problem with HLHS. The problem might be subendocardial ischemia for all we know, much like ischemic mitral valve regurgitation. So, can you find some positive elements for us, that is, improvement in survival, for example, or better freedom from transplantation, in the patients who underwent TV repair? Are we actually doing something good for these patients when we repair the TV?

Dr Bautista-Hernandez. That is an excellent point, but we did not compare patients who did not undergo TV repair in the present study, so I cannot really answer your question.

Dr Bacha. But you know that group of patients very well. Do you have an impression, at least, compared with the non-tricuspid valvuloplasty patients?

Dr Bautista-Hernandez. Those who did not undergo TV repair and had significant TR, you mean?

Dr Bacha. Yes, or even those who did not have much TR. I mean, do they have the same survival? Do we, perhaps, by repairing the TVs, not affect the RV function, but their survival then equals those who never had TR? Do we achieve that by repairing their TVs?

Dr Bautista-Hernandez. My impression is that survival improves. We also had a presentation at the American Heart Association meeting in 2006, with preliminary data from Boston. We were able to compare 2 groups of patients with HLHS and significant TR, those who had undergone tricuspid valvuloplasty and those who had not. We found that those patients who had undergone TV repair did better than those who had not but had significant TR; thus, tricuspid valvuloplasty improved the outcomes in that challenging population.

Dr Tara Karamlou (San Francisco, Calif). Two questions. One of my questions is, again, regarding the timing. Could you, based on your data, try to differentiate whether particular mechanisms of TR would improve solely by unloading at the Glenn procedure or were these all structural issues that required valvuloplasty or other reparative techniques? That is the first question.

Dr Bautista-Hernandez. We did not have many patients at the time of stage II, so we cannot really answer that question. We do not have enough patients to answer that question.

Dr Karamlou. It might be interesting to try to look at that as you gather more patients.

Dr Bautista-Hernandez. Sure.
Dr Karamlou. Then, the second question is, you again were delaying a number of these repairs until the Fontan. Do you think you would have seen an incremental benefit in RV function had you intervened on the TV earlier, say at the Glenn?

Dr Bautista-Hernandez. With respect to the RV function before the first repair, which was undertaken for most patients at stage III, we did not have patients with severe RV dysfunction. So, I do not think that just by waiting until stage III that a significant worsening in RV function occurs. However, in our series, we performed tricuspid valvuloplasty whenever necessary through staged palliation.

Dr Christopher A. Caldarone (Toronto, Ontario, Canada). That was a very nice presentation. If you do not mind, could you just tell us a little bit more about the relationship between the anterior leaflet prolapse and the anterior PM repositioning. You identified anterior leaflet prolapse as an important indicator of a bad actor, and I think that is a very important observation. You also stated that anterior PM repositioning was effective in your analysis.

First, could you actually conclude that anterior PM repositioning was statistically important?

Second, what was the relationship of anterior PM repositioning with regard to the subset of patients with anterior leaflet prolapse?

Dr Bautista-Hernandez. That is a very good question. This series goes from 2000 to 2012; thus, PM repositioning is a technique we have recently introduced into our clinical armamentarium. Therefore, it was not being performed at the beginning of our study. At that time, we were mainly using AS commissuroplasty. Moreover, we had just a few patients with PM repositioning in our series; thus, we cannot ensure that it is an effective technique. In addition, we do not have long-term follow-up data for those patients, so we must be cautious about sending out the message that PM repositioning is an outstanding technique.

Dr Caldarone. Well, it might very well turn out to be. Among all the techniques you have described, anterior PM repositioning is the only technique that manages the subvalvular support rather than just the level of the annulus and the leaflet. So I think it probably will be very important. But I just wanted to see whether you could actually state that you have proved the point, and it sounds like it is premature to do so.

Dr Bautista-Hernandez. It is premature. But our last conclusion was exactly that. Perhaps with this approach we can treat, not only the annulus, but also the subvalvular apparatus and, therefore, approach patients with a tailored surgical repair. I mean that you study the patient preoperatively and decide which technique will be a better fit.

Dr Carl L. Backer (Chicago, Ill). When you perform a valvuloplasty, are you closing the commissure between the anterior and septal leaflets with interrupted sutures?

Dr Bautista-Hernandez. The commissuroplasty you mean?

Dr Backer. Yes.

Dr Bautista-Hernandez. Yes.

Dr Backer. Let me poll the audience. I have always used the posterior leaflet obliteration technique described by the Michigan group. Let us take a vote and see how many surgeons are primarily using that posterior leaflet obliteration technique.

(Show of hands.)

I see about 10 hands. 
How many surgeons are using AS commissuroplasty, as per the Boston strategy?

(Show of hands.)

That looks like about 50 to 60 hands.

How many people are routinely placing an annuloplasty, some sort of an annuloplasty, when they operate on these patients?

(Show of hands.)

I see about 20 hands.

And, how many surgeons rarely, if ever, use annuloplasty?
(Show of hands.)

It looks like about 40 hands. It appears we do not have this problem completely figured out. We need to keep working on it.

Dr Hirsch-Romano. And how many people are using rings?

(Show of hands.)

Dr Hirsch-Romano. So 1.

So Dr Del Nido, you have your work cut out for you.

Dr Backer. Perhaps next year's presidential address. 